\section{Prevalência de cárie dentária em pré-escolares da rede pública de Recife, Pernambuco, Brasil, aos quatro anos de idade}

\author{
Caries prevalence in 4-year-old preschoolers \\ attending public schools in Recife, \\ Pernambuco, Brazil
}

\author{
1 Faculdade de Odontologia \\ de Pernambuco, \\ Universidade de \\ Pernambuco, Recife, Brasil. \\ Correspondência \\ Sandra Feitosa \\ Rua Pastor José Amaro \\ da Silva 112/701, Recife, PE \\ 51021-230, Brasil. \\ shfeitosa@zipmail.com.br
}

\begin{abstract}
The aim of this study was to determine caries prevalence in 4-year-old preschoolers of both sexes in municipal schools in Recife, Pernambuco, Brazil, in 2002, emphasizing their needs assessment. Data were collected by examining 861 preschoolers from 45 municipal schools. Examination was conducted by one examiner (Kappa = 1) in the school and after parental consent forms were obtained. Dental caries was recorded using the dmf-t (decayed, missing, and filled teeth) index. Data analysis showed caries prevalence of 47.00\% and dmf-t of 2.06. Only 6.40\% of the children had their teeth treated. The examined children showed high dental caries prevalence (47.00\%), despite a low prevalence of severe caries (8.94\%).
\end{abstract}

Dental Caries; Prevalence; Oral Health;Child
Sandra Feitosa 1

Viviane Colares 1

\section{Introdução}

O controle e prevenção da cárie dentária é um desafio constante para pesquisadores em todo o mundo. A adoção de medidas preventivas democráticas de controle da cárie dentária como a fluoretação da água de abastecimento e dos cremes dentais, permitiram uma melhora substancial na saúde bucal de diferentes grupos sociais 1,2. Porém, uma significativa parcela da população ainda sofre de problemas odontológicos 2 .

A prevalência da cárie dentária no Brasil em crianças jovens vem sendo estudada ao longo dos anos. Bönecker et al. 3 verificaram que crianças residentes em Diadema, São Paulo, com idade variando entre 30 e 36 meses, que participaram da Campanha Nacional de Multivacinação, apresentaram um ceo-d médio de 2,89 . Esses autores afirmaram que o grau de severidade da cárie dentária em crianças na faixa etária de 0 a 36 meses aumenta proporcionalmente ao aumento da idade. No Norte do Brasil, Arias et al. 4, com o objetivo de caracterizar a saúde oral de crianças de 0 a 36 meses, encontraram uma prevalência de $6,28 \%$ de dentes cavitados em crianças pertencentes a creches públicas do Município de Belém, Pará. Leite \& Ribeiro 5, avaliando a prevalência de cárie em crianças com 2 a 6 anos de idade, de baixo nível sócio-econômico, assistidos em creches públicas da cidade de Juiz de Fora, Minas Gerais, encontraram um ceo-d médio de 2,03 e 
verificaram que $50,60 \%$ das crianças estudadas encontravam-se livres de cárie.

A polarização da cárie dentária, fenômeno onde a prevalência da patologia acomete, com maior freqüência, grupos sociais menos favorecidos sócio-economicamente, fortaleceu mais intensamente as preocupações no controle e prevenção da cárie em comunidades carentes, bem como as repercussões da cárie dentária na vida do paciente infantil.

Este trabalho teve como objetivo determinar a prevalência da cárie dentária em crianças com quatro anos de idade, de ambos os sexos, pertencentes a escolas municipais da cidade do Recife, Pernambuco, no ano de 2002, enfatizando a necessidade de tratamento nesse grupo etário. Este trabalho faz parte de uma pesquisa maior de dissertação de Mestrado na área de concentração em Odontopediatria da Faculdade de Odontologia da Universidade de Pernambuco.

\section{Revista da literatura}

\section{- Prevalência de cárie dentária}

A modalidade de estudo epidemiológico que melhor permite conhecer o perfil de uma situação dentro de uma comunidade, de maneira rápida e com baixo custo, é o estudo de prevalência ou também denominado transversal ou seccional. $\mathrm{O}$ estudo de prevalência tem o objetivo de fornecer a medição de problemas em um determinado objeto de pesquisa, permitindo analisar variáveis como a distribuição de doenças por idade, sexo, etnia. Esse tipo de es- tudo é muito útil como base de planejamento e de determinação de necessidades coletivas de tratamento 6,7 .

Diversos trabalhos têm investigado os índices de cárie dentária, nas mais diversas localidades, em crianças jovens no Brasil. A Tabela 1 apresenta alguns estudos desenvolvidos nos últimos anos em diferentes regiões do país.

Deve-se salientar que, de acordo com Nadanovsky 2, mesmo com o crescente declínio da cárie dentária em diversos locais e em determinados grupos etários, uma significativa parcela da população ainda sofre de problemas odontológicos, onde $70,00 \%$ dos dentes cariados estão em $30,00 \%$ das crianças.

Os fatores sócio-econômicos têm recebido considerável atenção na literatura científica, como forte fator de risco à saúde. De acordo com Pereira 6, a associação entre a renda, por exemplo, é muito nítida, tanto em nível individual quanto no coletivo. Nas famílias de menor renda, especialmente nos países do Terceiro Mundo, encontra-se uma alta freqüência de desnutrição, doenças sexualmente transmissíveis e condições ambientais deficientes.

Na literatura odontológica, a associação entre nível sócio-econômico e cárie dentária está amplamente estabelecida 9,10,11,12,13,14,15.

Em 2001, Grillcrist et al. 14 realizaram uma pesquisa com o objetivo de investigar a relação entre o status sócio-econômico da comunidade e a saúde oral da criança. O estudo foi desenvolvido em 62 comunidades do Tennessee, Estados Unidos, onde foram examinadas 17.256 crianças, com idade variando entre 5 e 11 anos. Os autores concluíram que as crianças perten-

Prevalência de cárie dentária na população infantil brasileira.

\begin{tabular}{|c|c|c|c|c|c|}
\hline Autor (es) & Ano & Local & $\begin{array}{l}\text { Idade } \\
\text { da criança } \\
\text { (meses) }\end{array}$ & $\begin{array}{l}\text { Prevalência } \\
\text { de cárie (\% de } \\
\text { crianças afetadas) }\end{array}$ & $\begin{array}{l}\text { ceo-d } \\
\text { médio }\end{array}$ \\
\hline Mattos-Graner et al. 19 & 1996 & Piracicaba, São Paulo & $6-36$ & 23,60 & - \\
\hline Bönecker et al. 3 & 1997 & Diadema, São Paulo & $30-36$ & - & 2,89 \\
\hline Arias et al. 4 & 1997 & Belém, Pará & $30-36$ & 43,94 & - \\
\hline Leite \& Ribeiro 5 & 2000 & Juiz de Fora, Minas Gerais & $24-72$ & - & 2,03 \\
\hline Dini et al. 21 & 2000 & Araraquara, São Paulo & $36-48$ & 46,00 & - \\
\hline Leme 20 & 2001 & Brasília, Distrito Federal & $36-71$ & 36,04 & - \\
\hline Rosenblatt \& Zarzar 22 & 2002 & Recife, Pernambuco & $12-36$ & 28,46 & 3,62 \\
\hline
\end{tabular}

Fonte: Feitosa 8 . 
centes ao grupo sócio-econômico menos favorecido apresentaram pior saúde oral quando comparadas ao grupo mais favorecido. Essas crianças com saúde oral prejudicada tinham uma maior experiência de cárie, maior necessidade de tratamento, uma maior prevalência de trauma nos incisivos e menor prevalência de selantes oclusais.

\section{Metodologia}

Este estudo transversal foi desenvolvido na cidade do Recife, capital do Estado de Pernambuco. A cidade está dividida em seis regiões político-administrativas possuindo 144 escolas com educação infantil em todo o município, onde estavam matriculados 5.093 alunos, com quatro anos de idade, de ambos os sexos, no ano de 2002

Para a determinação do tamanho amostral foi realizado um estudo piloto a fim de estabelecer a prevalência da doença na população. A prevalência de cárie dentária encontrada foi de $48,30 \%$ e de cárie severa, $8,00 \%$, numa amostra de 101 crianças distribuídas em quatro escolas de diferentes regiões da cidade.

Foram considerados erro de $2,00 \%$, para mais ou para menos, na obtenção da prevalência de cárie severa, e confiabilidade de 95,00\% de que o erro não seja ultrapassado. A fórmula utilizada para determinação do tamanho amostral considerou um tamanho populacional infinito (população grande) 16.

Com o objetivo de minimizar possíveis perdas durante a operação de levantamento dos dados, o que poderia comprometer a representatividade da amostra, decidiu-se aumentar o tamanho amostral em $20,00 \% 6$, resultando em 850 crianças.

A amostra foi estratificada proporcionalmente ao número de alunos em cada região político-administratitva. Foi estabelecido, então, o número de escolas em cada região políticoadministratitva que seriam incluídas na pesquisa, e posterior sorteio dessas escolas 17. Das 144 pertencentes à cidade de Recife, foi selecionada uma amostra de 45 escolas.

Participaram da pesquisa crianças com quatro anos de idade, mental e fisicamente normais, cujos responsáveis assinaram o consentimento livre e esclarecido. Essas crianças pertenciam a famílias de baixo nível sócio-econômico, onde seus responsáveis recebiam, em média, um salário mínimo de renda mensal.

Foi realizado exame clínico da cavidade bucal por uma pesquisadora calibrada, na própria escola, sob os critérios do Ministério da Saúde 18.
O procedimento clínico foi realizado na sala de aula, sob a luz artificial, na cadeira escolar, utilizando toda a vestimenta necessária e material descartável, incluindo abaixadores de língua.

Para calibração da pesquisadora durante o exame clínico foi adotado o seguinte procedimento: a cada dez crianças, uma foi reexaminada após 24 horas da realização do exame. A seleção dessa criança deu-se de forma aleatória entre os menores examinados. Ao término da coleta, 86 crianças foram reexaminadas, obtendo-se um Kappa = 1. Essa calibração considerada perfeita, deveu-se ao critério objetivo adotado para a cárie dentária, relatado abaixo.

Para determinar a prevalência de cárie dentária foi utilizado o índice ceo-d. O índice ceod é o correspondente ao CPO-D em relação à dentição decídua, incluindo os dentes cariados (c), com extração indicada (e) e obturados (o), excluindo os extraídos devido às dificuldades em separar os que o foram por causa de cárie, dos perdidos pelo processo natural de esfoliação dentária 7. Os elementos dentários indicados para exodontia apresentavam-se com destruição coronária extensa, com a presença de restos radiculares.

Os dados desta pesquisa foram processados em banco de dados com as variáveis devidamente categorizadas, no programa SPSS (Statistical Package for Social Science) versão 11.0, com digitação única, propiciando à pesquisa uma maior fidedignidade e confiabilidade. Além da quantidade de dentes cariados, perdidos e obturados (todos por cárie - ceo-d), foram obtidas as medidas de tendência central: média, mediana e desvio padrão.

Este estudo foi aprovado pelo Comitê de Ética e Pesquisa da Universidade de Pernambuco, sob o protocolo no E024/02, em 20 de fevereiro de 2002.

\section{Resultados}

Após a análise dos dados coletados, verificou-se que das 861 crianças examinadas, 449 (52,10\%) eram do sexo masculino e $412(47,90 \%)$ do feminino.

A Figura 1 representa as crianças com e sem experiência de cárie.

Do total de crianças examinadas, $27(3,10 \%)$ apresentavam um ou mais dentes indicados para extração. Do total de 405 crianças que apresentavam cárie, apenas 55 (13,60\%) haviam recebido algum tipo de tratamento restaurador. O ceo-d médio encontrado nessa população foi de 2,06.

Um percentual de $8,94 \%$ das crianças portadoras de cárie dentária apresentavam a doen- 
ça num grau severo, com cárie nos quatro quadrantes, comprometimento de, no mínimo, 1/3 da coroa de qualquer incisivo e quatro molares com lesão de cárie clinicamente visível.

A Tabela 2 apresenta os dados do ceo-d em forma esquemática.

\section{Discussão}

Os estudos transversais vêm sendo utilizados cada vez mais pelos pesquisadores $5,19,20$, pois trata-se de um tipo de estudo de baixo custo e que permite traçar um perfil da comunidade estudada em um curto espaço de tempo 6,7.

As prevalências de cárie encontradas nos diferentes estudos variam entre localidades e entre grupos etários 2. Dini et al. 21 encontraram um prevalência de cárie de $46,00 \%$ e ceo-d médio de 2,03 em crianças de três a quatro anos residentes em Araraquara, São Paulo. Dados semelhantes foram observados por Leite \& Ribeiro 5 ao examinarem crianças de dois a seis anos em Juiz de Fora, Minas Gerais (ceo-d médio de 2,03). Esses resultados coincidem com aqueles verificados neste estudo, no entanto, Leme 20 observou uma menor prevalência de cárie $(36,04 \%)$ ao investigar crianças de três a quatro anos em Brasília, Distrito Federal.

Rosenblatt \& Zarzar 22, em pesquisa realizada em Recife, entre crianças com idade variando de um a três anos, encontraram uma prevalência de cárie precoce na infância de $28,46 \%$. Considerando a maior prevalência encontrada neste estudo de $47,00 \%$ em crianças com quatro anos, corroboram-se os estudos de O'Sullivan \& Tinanoff 23 e Rosenblatt \& Zarzar 22 , tendo a prevalência de cárie aumentado com a idade.

As diferenças percentuais encontradas nas pesquisas de prevalências justificam-se pela falta de padronização nos critérios de diagnóstico acerca da cárie dentária. No presente estudo, foi considerado cárie a presença de cavitação no elemento dentário, de acordo com Fejerskov 24. Optou-se, ainda, pela utilização de espátulas de madeira para realização do exame clínico nas escolas 18.

Apesar do alto percentual de cárie dentária $(47,00 \%)$ na população estudada, o ceo-d médio foi de 2,06; porém com desvio-padrão de 3,00 , o que indica que segmentos dentro do grupo possuem altos valores de ceo-d. Observa-se, dessa forma, uma concentração da doença dentro do grupo sócio-econômico menos favorecido.

Considerando a não fluoretação das águas de abastecimento da cidade de Recife, existem alguns pontos a serem discutidos, como a presença de defeitos na estrutura do esmalte dental, tornando o elemento dentário mais susceptível ao desenvolvimento da patologia.

Um dado preocupante foi o percentual encontrado de crianças que possuíam dentes obturados $(13,60 \%)$, o que corresponde a 55 crian-

Figura 1

Distribuição das crianças estudadas de acordo com a prevalência de cárie.

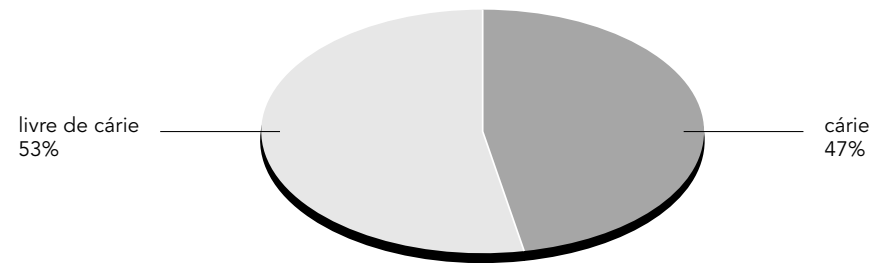

Tabela 2

Distribuição das medidas de tendência central, desvio-padrão e porcentual de ceo-d, segundo seus elementos constituintes.

\begin{tabular}{lcccrrr}
\hline $\begin{array}{l}\text { ceo-d e elementos } \\
\text { constituintes }\end{array}$ & Mínimo & Máximo & Média & Mediana & Desvio padrão ceo-d (\%) \\
\hline Cariados & 0 & 20 & 1,90 & 0,00 & 2,89 & 92,30 \\
Indicados para extração & 0 & 10 & 0,05 & 0,00 & 0,34 & 2,40 \\
Obturados & 0 & 10 & 0,11 & 0,00 & 0,49 & 3,30 \\
ceo-d & 0 & 20 & 2,06 & 0,00 & 3,00 & 100,00
\end{tabular}


ças entre as 405 que apresentavam cárie. Considerando que essas crianças pertenciam ao grupo com menor poder aquisitivo, esses achados nos remetem aos relatos de Gillcrist et al. 14, quando afirmaram que crianças dessa classe social têm maior experiência de cárie e, conseqüentemente, maior necessidade de tratamento dentário.

A falta de tratamento desse grupo estudado pode ser devido ao fato de se tratar de crianças em idade pré-escolar, possuindo exclusivamente dentes decíduos, em geral menos valorizados por pais e profissionais. Sugere-se que outros estudos sejam desenvolvidos visando a investigar a saúde bucal das crianças com dentição decídua no Brasil, assim como melhor avaliar a percepção pelos responsáveis e dentistas. A faixa etária estudada representa, em geral, um desafio para os profissionais com relação ao controle do comportamento, o que também pode interferir na oferta de atenção odontológica para esse grupo de crianças.

Resumo

Esta pesquisa teve como objetivo determinar a prevalência de cárie dentária em crianças com quatro anos de idade, de ambos os sexos, das escolas públicas municipais da cidade do Recife, Pernambuco, Brasil,no ano de 2002, enfatizando a necessidade de tratamento dentário para essa faixa etária. Os dados foram coletados por meio do exame clínico de 861 crianças pertencentes a 45 escolas públicas. O exame clínico foi realizado por uma examinadora calibrada (Kappa $=1$ ), na própria escola, após assinatura do consentimento livre e esclarecido pelo responsável. Para determinação da prevalência de cárie dentária foi adotado o índice ceo-d. Após análise dos dados, verificou-se que a prevalência de cárie dentária na população estudada foi de 47,00\% e o ceo-d médio foi de 2,06. Apenas $13,60 \%$ das crianças que apresentavam cárie possuíam restaurações. É pertinente concluir que as crianças examinadas apresentaram uma alta prevalência de cárie dentária (47,00\%), apesar do percentual de crianças portadoras de cárie em estágio severo ter sido considerado baixo (8,94\%).

Cárie Dentária; Prevalência; Saúde Bucal;Criança
Considerando o baixo percentual de crianças portadoras de cárie em um grau severo $(8,90 \%)$, sugere-se que, para esse grupo minoritário, seja oferecido atendimento odontológico sob sedação com menor número de consultas, de forma eficiente, nos centros públicos de saúde que são referência em Odontopediatria.

\section{Conclusão}

Após a análise dos dados coletados nesta pesquisa, é pertinente concluir que:

- as crianças das escolas públicas municipais da cidade do Recife apresentam uma alta prevalência de cárie dentária $(47,00 \%)$;

- apesar da prevalência de cárie ter sido alta, o percentual de crianças portadoras de cárie severa foi considerado baixo $(8,94 \%)$;

- entre as crianças que apresentavam cárie, um baixo percentual $(13,60 \%)$ recebeu algum tipo de tratamento curativo.

\section{Colaboradores}

S. Feitosa realizou a coleta dos dados do estudo. A análise dos dados e a redação do texto foram realizadas por ambas as autoras. 


\section{Referências}

1. Marcenes W, Bönecker MJS. Aspectos epidemiológicos e sociais das doenças bucais. In: Buischi YP, organizador. Promoção de saúde bucal na clínica odontológica. São Paulo: Artes Médicas; 2000. p. 75-98.

2. Nadanovsky P. O declínio da cárie dentária. In: Pinto VG, organizador. Saúde bucal coletiva. São Paulo: Santos; 2000. p. 341-51.

3. Bönecker MJS, Guedes-Pinto AC, Walter LRF. Prevalência, distribuição e grau de afecção de cárie dentária em crianças de 0 a 36 meses de idade. Revista da Associação Paulista de Odontologia 1997; 51:535-40.

4. Arias SMB, Brandão AMM, Nogueira AJS. Prevalência de cárie em bebês de 0-3 anos. RGO 1997; 45:163-9.

5. Leite ICG, Ribeiro RA. Dental caries in the primary dentition in public nursery school children in Juiz de Fora, Minas Gerais, Brasil. Cad Saúde Pública 2000; 16:717-22.

6. Pereira MG. Epidemiologia - teoria e prática. Rio de Janeiro: Guanabara Koogan; 1995.

7. Pinto VG. Identificação de problemas. In: Pinto VG, organizador. Saúde bucal coletiva. São Paulo: Santos; 2000. p. 139-222.

8. Feitosa S. As repercussões psicossociais da cárie severa em crianças com 4 anos de idade da Cidade de Recife [Dissertação de Mestrado]. Camaragibe: Faculdade de Odontologia, Universidade de Pernambuco; 2003.

9. Reisine S, Litt M. Social and psychological theories and their use for dental pratice. Int Dent J 1993; 43(3 Suppl 1):279-87.

10. Gratix D, Holloway PJ. Factors of deprivation associated with dental caries in young children. Community Den Health 1994; 11:66-70.

11. Litt M, Reisine S, Tinanoff N. Multidimensional causal model of dental caries development lowincome preschol children. Public Health Rep 1995; 57:607-17.

12. Tomita NE, Bijella VT, Lopes ES, Franco LJ. Prevalência de cárie dentária em crianças da faixa etária de 0 a 6 anos matriculadas em creches: importância de fatores socioeconômicos. Rev Saúde Pública 1996; 30:413-20.
13. Reisine S, Douglas JM. Psychosocial and behavioral issues in early childhood caries. Community Dent Oral Epidemiol 1998; 26(1 Suppl):32-44.

14. Gillcrist JA, Brumley DE, Blackford JU. Community socioeconomic status and children's dental health. J Am Dent Assoc 2001; 132:216-22.

15. Marques AMBC. Perfil sociocultural de escolares livres de cárie aos 5 anos na Cidade do Recife [Dissertação de Mestrado]. Camaragibe: Faculdade de Odontologia, Universidade de Pernambuco; 2001.

16. Zar JH. Biostatistical analysis. New Jersey: Prentice Hall; 1999.

17. Cochran WG. Sampling techiniques New York: John Wiley \& Sons; 1977.

18. Secretaria da Assistência à Saúde. Programa nacional de doenças transmissíveis. Aids e herpes na prática odontológica. Brasília: Ministério da Saúde; 1994.

19. Mattos-Graner RO, Rontani RMP, Gavião MBD, Bocatto HARC. Caries prevalence in 6-36-monthold Brazilian children. Community Dent Health 1996; 13:96-8.

20. Leme TDP. Prevalência de cárie severa em pré-escolares de 36 a 71 meses de idade. Considerações sobre hábitos alimentares [Dissertação de Mestrado]. Brasília: Universidade de Brasília; 2001.

21. Dini EL, Holt RD, Bedi R. Caries and its association with infant feeding and oral health-related behaviours in 3-4-year-old Brazilian children. Community Dent Oral Epidemiol 2000; 28:241-8.

22. Rosenblatt A, Zarzar P. The prevalence of early childhood caries in 12-to 36-month-old children in Recife, Brazil. ASCD J Dent Child 2002; 2:1-6.

23. O'Sullivan DM, Tinanoff N. The association of early dental caries patterns with caries incidence in preschool children. J Public Health Dent 1996; 56:81-3.

24. Fejerskov O. Concepts of dental caries and their consequences for understanding the disease. Community Dent Oral Epidemiol 1997; 25:5-12.

Recebido em 15/Mai/2003

Versão final reapresentada em 21/Out/2003

Aprovado em 23/Out/2003 